
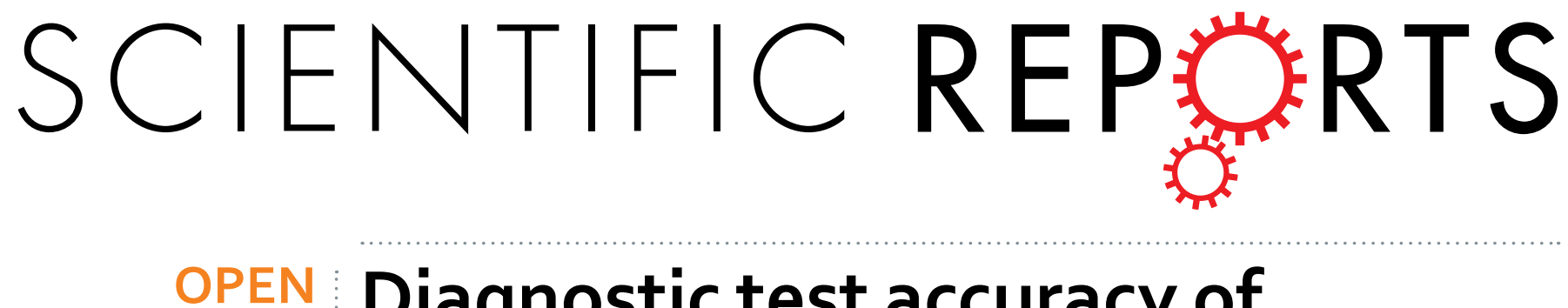

\title{
Diagnostic test accuracy of glutamate dehydrogenase for Clostridium difficile: Systematic
}

Received: 05 April 2016

Accepted: 22 June 2016

Published: 15 July 2016

\section{review and meta-analysis}

Jun Arimoto ${ }^{1}$, Nobuyuki Horita ${ }^{2}$, Shingo Kato ${ }^{1}$, Akiko Fuyuki ${ }^{1}$, Takuma Higurashi ${ }^{1}$, Hidenori Ohkubo ${ }^{1}$, Hiroki Endo ${ }^{1}$, Nonaka Takashi ${ }^{1}$, Takeshi Kaneko ${ }^{2}$ \& Atsushi Nakajima ${ }^{1}$

We performed this systematic review and meta-analysis to assess the diagnostic accuracy of detecting glutamate dehydrogenase (GDH) for Clostridium difficile infection (CDI) based on the hierarchical model. Two investigators electrically searched four databases. Reference tests were stool cell cytotoxicity neutralization assay (CCNA) and stool toxigenic culture (TC). To assess the overall accuracy, we calculated the diagnostic odds ratio (DOR) using a DerSimonian-Laird random-model and area the under hierarchical summary receiver operating characteristics (AUC) using Holling's proportional hazard models. The summary estimate of the sensitivity and the specificity were obtained using the bivariate model. According to 42 reports consisting of 3055 reference positive comparisons, and 26188 reference negative comparisons, the DOR was $115\left(95 \% \mathrm{Cl}: 77-172, \mathrm{I}^{2}=12.0 \%\right)$ and the $\mathrm{AUC}$ was $0.970(95 \% \mathrm{Cl}$ : $0.958-0.982$ ). The summary estimate of sensitivity and specificity were 0.911 ( $95 \% \mathrm{Cl}: 0.871-0.940)$ and $0.912(95 \% \mathrm{Cl}: 0.892-0.928)$. The positive and negative likelihood ratios were $10.4(95 \% \mathrm{Cl} 8.4-12.7)$ and $0.098(95 \% \mathrm{Cl} 0.066-0.142)$, respectively. Detecting GDH for the diagnosis of CDI had both high sensitivity and specificity. Considering its low cost and prevalence, it is appropriate for a screening test for CDI.

Clostridium difficile is an anaerobic, spore-forming Gram-positive bacillus that is capable of causing diarrhea mediated by the production of C. difficile toxins $\mathrm{A}$ and $\mathrm{B}^{1}$. C. difficile infection (CDI) accounts for $15 \%$ to $25 \%$ of antibiotic-associated diarrhea ${ }^{2}$. The two serious risk factors of CDI are exposure to antibiotics exposure to the organism, usually during a hospital stay. Others factors are older age, gastrointestinal tract surgery, and anti-acid medications including proton-pump inhibitors ${ }^{3,4}$. The severity of CDI ranges from very mild to toxic megacolon with septic shock. Metronidazole and vancomycin are the most frequently used first-line antibiotics to treat CDI. Fecal microbiota transplantation has recently been proposed as alternative treatment ${ }^{5,6}$. However, patients who do not respond to these medications may require intensive care or colectomy. According to surveillance, mortality from CDI is approximately $5.7 \%$.

The initial step in proper treatment of CDI is quick and accurate diagnosis of CDI. However, none of the existing C. difficile examinations is perfect in view of accuracy, cost, and incubation time ${ }^{8-11}$. Nucleic acid amplification tests (NAATs) such as polymerase chain reaction and loop-mediated isothermal amplification provide quick and accurate diagnosis ${ }^{12-14}$, albeit a high cost. Though expensive, single-step diagnosis strategies utilizing only a NAAT is the simplest diagnosis strategy ${ }^{8}$. Multiple-step diagnosis is another strategy for which low cost exam, namely glutamate dehydrogenase (GDH) assay, is used as the first-step tool, followed by NAATs or by toxin tests only for specimens with positive result in the first test ${ }^{8}$. Detecting GDH seems a reasonable screening tool because this non-expensive and non-time-consuming test is sensitive ${ }^{15}$.

Since the last decade, an increasing number of observational studies concerning GDH assay accuracy for C. difficile detection have been reported ${ }^{15}$. The current understanding is that single-step GDH assay could not confirm the CDI. Nonetheless, evaluation of the single-step GDH assay is necessary for some reasons. Single-step GDH assay negative usually warrants CDI negative. In addition, we had to know the diagnostic test accuracy of

${ }^{1}$ Department of Gastroenterology and Hepatology, Yokohama City University School of Medicine, Yokohama, Japan. ${ }^{2}$ Department of Pulmonology, Yokohama City University Graduate School of Medicine, Yokohama, Japan. Correspondence and requests for materials should be addressed to N.H. (email: horitano@yokohama-cu.ac.jp) 
single-step GDH assay to design two-step and three-step GDH assays. Shetty et al. reported a systematic review concerning this topic in $2011^{15}$. However, due to considerable heterogeneity among studies, their study mainly focused on describing the summary receiver operating characteristic (SROC) curve and avoided presenting accurate pooled sensitivity and specificity. They avoided it because univariate meta-analysis leads to gross underestimates of sensitivity and specificity when the diagnostic test performance differs owing to local conditions ${ }^{15}$. Even though GDH is commonly accepted as a screening tool for CDI, no published meta-analysis has provided straightforward summary estimates of sensitivity and specificity of GHD to diagnose CDI. The recent meta-analysis methodology for diagnostic test accuracy strongly recommends use of a hierarchical model, which enables us appropriately deal with the tradeoff between sensitivity and specificity caused by the threshold effect ${ }^{16-19}$. In addition, many original studies have been published concerning GDH since the review by Shetty et al. was published. Thus, we believe an updated systematic review and meta-analysis using a hierarchical model is required to reveal how accurate the GDH assay is in diagnosing CDI.

\section{Methods}

Study registration. The protocol has been registered with the international prospective register of systematic reviews (PROSPERO) as number CRD4201603276020. This study protocol follows the Preferred Reporting Items for Systematic Reviews and Meta-Analyses (PRISMA) statement and the Cochrane Handbook for Diagnostic Test Accuracy Reviews ${ }^{16,21}$. Institutional review board approval and patient consent were waivered because of the review nature of this study.

Eligibility criteria. Type of studies. We had planned to include both two-gate cohort studies and one-gate case-control studies. However, we eventually found no case-control study. We included a study with sufficient data to estimate the sensitivity and the specificity of GDH assay for CDI using PCR as reference standard. Along with a study with single-step GDH assay, we included a study that evaluated multi-step GDH assay when we could extract the separate GDH data from such study. Conference abstracts, short articles, and non-full articles were allowed.

Participants. Meta-analysis was conducted based on numbers of specimens but not on numbers of persons. Specimens from cases with a possible diagnosis of CDI, diarrheal stool, and liquid stood were preferred. When a study included formed specimens, we marked a high applicability concern for patient selection ${ }^{22}$. Human non-stool samples, animal stool samples, and food samples were excluded.

Index test. As an index test, we included any stool GDH assay including commercialized kit and in-house assays.

Reference test. The stool cell cytotoxicity neutralization assay (CCNA) and stool toxigenic culture (TC) were used as reference tests ${ }^{8}$. Other tests such as NAATs, and simple culture were not regarded as references in this study.

Outcome. First, we made a two by two contingency from the numbers of true positives/false negatives/false positives/true negatives presented in each original study. Then, we assessed the diagnostic odds ratio (DOR), and the area under the hierarchical SROC curve (AUC) to find the overall accuracy. The summary estimate of sensitivity, specificity, positive likelihood ratio (PLR), negative likelihood ratio (NLR), positive predictive value (PPV), and negative predictive value (NPV) were also assessed ${ }^{16}$.

Literature search strategy. We had conducted a database search using PubMed, Embase, the Cochrane Library, and Web of Science on January $5^{\text {th }}, 2016$. Search formulas were presented in Supplementary Text 1.

References to previously published reviews and those of included original studies were hand-searched.

Study selection. The two investigators independently conducted title/abstract screening after uploading a citation list into the software, Endnote X7 (THOMSON REUTERS, Philadelphia, USA). Articles that were not excluded by at least one investigator were passed for scrutiny. We scrutinized them by checking the full text independently. The final inclusion was determined after discussion to solve any discrepancies. Duplicate use of the same data was carefully excluded.

Data extraction. The two investigators independently extracted data and input them into Microsoft Excel 2013. Then, the data extracted by the two investigators were crosschecked. Discrepancies were resolved by discussion between the two investigators.

Quality assessment for bias and applicability. The two investigators independently evaluated each study. Seven domains of A Revised Tool for the Quality Assessment of Diagnostic Accuracy Studies (QUADAS-2) evaluation sheet were scored ${ }^{22}$. If the two investigators gave different scores, the discrepancies were resolved through discussion.

For the current systematic review, we assessed the quality using the following principles. Excluding patients for whom the authors had difficulty judging whether the patients had CDI or not was a reason for a high risk of patient selection bias. No description of consecutiveness and randomness was a reason for an unclear risk of patient selection. Including formed stool was a reason for a high patient selection applicability concern. Risk of bias for index and reference tests was generally not suspected because we can judge the results of GDH, CCNA, and TC unbiasedly. Bias in flow and timing was also not suspected because both index and reference tests were conducted on the same stool specimen.

A study without high risk of bias and high applicability concerns was regarded as a non-high-risk study. 
Statistical analysis and quantitative synthesis. Data synthesis. When, two GDH assays were compared to a reference test in a report, one assay was selected in the following order: Chek-60, Quik Chek, Culturette followed by Triage. This order was decided based on a number of studies that assessed each assay and a number of patients that were assessed for each assay. Data from two index assays in a study were independently used for index-test-based subgroup analysis. Similarly, when both CCNA and TC were used as references in a report, we chose CCNA as a reference test because recent study suggested that CCNA is more reliable than TC ${ }^{23}$. Data from two reference tests in a study were independently used for reference-test-based subgroup analysis.

We used both hierarchical SROC curves and bivariate models ${ }^{16-19}$. To assess the overall accuracy, we calculated the DOR using a DerSimonian-Laird random-model and the AUC using Holling's proportional hazard models $^{24,25}$. According to a criterion of Jones et al. AUC $>0.97,0.93-0.96,0.75-0.92$, and $0.5-0.75$ were interpreted as "excellent," "very good," "good," and "reasonable," respectively ${ }^{26}$. A paired forest plot, hierarchical SROC curve, and the summary estimate of the sensitivity and the specificity were obtained using the bivariate model ${ }^{16}$. PLR and NLR were obtained from summary estimates of sensitivity and specificity. According to Grimes et al. PLR in the range of 2-5,5-10, and $>10$ represent small, moderate, and large increases of probability when the test is positive. Similarly, NLR in the range of $0.2-0.5,0.2-0.1$, and $<0.1$ represent small, moderate, and large decreases of probability when the test is negative ${ }^{27}$. We also obtained PPV and NPV, which were calculated from summary estimates of sensitivity and specificity, as variables depending on pretest probability ranging from 0 to $100 \%$.

As a sensitivity analysis, we conducted subgroup analysis including only non-high-risk studies and subgroup analysis based on reference tests. In addition, to compare the diagnostic accuracy, index-test-based subgroup analyses were carried out.

GRADE Evidence Profile table wad also presented ${ }^{28}$.

Heterogeneity. We used the $\mathrm{I}^{2}$ statistic to evaluate the heterogeneity of overall test accuracy among the studies: $0 \%$ meant no heterogeneity, $0 \%$ to $40 \%$ meant not important heterogeneity, $30 \%$ to $60 \%$ meant moderate heterogeneity, $50 \%$ to $90 \%$ meant substantial heterogeneity, $75 \%$ to $100 \%$ meant considerable heterogeneity ${ }^{29}$.

Software. A paired forest plot was made using Reviewing Manager ver. 5.3 (Cochrane Collaboration, Oxford, UK). The following commands of the "mada" package in the free software R were used: "madauni" for DOR, "phm" for AUC, and "reitsma" for the hierarchical SROC curve and a summary estimate for the sensitivity and the specificity ${ }^{24,25}$. GRADE evidence profile table was output from GRADE website ${ }^{30}$.

\section{Results}

Study search. Of 684 articles that met the preliminary criteria, 304, 213, and 125 were excluded through removal of duplication, title/abstract screening, and full-article scrutinization, respectively (Supplementary Figure 1). We finally found 42 eligible reports (Table 1, Supplementary Text 2). All the 42 reports used the cohort study approach and we found no case-control study. The 42 reports comprised 33 full-length articles, seven conference abstracts, a conference poster, and a letter article, all of which were written in English. Among the 42, 17 were from the USA, six were from Canada, six were from the UK, and most of the others were from developed countries. Seven reports described comparisons of two index tests and five reports described comparisons of reference CCNA and TC, thus, we eventually evaluated 54 cohorts.

As a reference test, 31 used CCNA and 23 used TC. As an index test, 18 used Chek-60, 18 used Quik Chek, six used the Culturette Brand Latex Test, and five used Triage. The comparison between the index and the reference in each cohort ranged from 60 to 12365 with a median of 373 . The total number of comparisons was 47904, which consisted of 4946 reference positive comparisons and 42971 reference negative comparisons. Across the 54 cohorts, the sensitivity ranged from 0.23 to 1 with a median of 0.94 and the specificity ranged from 0.64 to 1 with a median of 0.92 (Fig. 1).

Among the 54 cohorts, 47 had high risk of flow and timing mostly due to duplicate use of multiple specimens from same patient. In addition, four had high risk of patient selection, three had high applicability concerns for patient selection, and one had high applicability concerns for the reference test (Supplementary Figure 2). Eventually six cohorts were classified as non-high-risk cohorts.

Diagnostic accuracy across all index tests. Using data from all 42 cohorts consisting of 3055 reference positive comparisons and 26188 reference negative comparisons, DOR was 115 (95\% confidence interval (95\% CI) $77-172, \mathrm{I}^{2}=12.0 \%$ ) and AUC was 0.970 (95\% CI 0.958-0.982) (Table 2, Fig. 2A). According to Jones' criteria, the AUC of 0.970 meant excellent overall diagnostic accuracy ${ }^{26}$. According to the first sensitivity analysis using data from 6 non-high-risk cohorts with 2745 comparisons, DOR was 189 (95\% CI 54-660, I ${ }^{2}=0 \%$ ) and AUC was 0.986 (95\% CI 0.976-0.998) (Table 2, Fig. 2B). For the second sensitivity analysis based on CCNA, DOR was 80 $\left(95 \%\right.$ CI $\left.50-131, \mathrm{I}^{2}=0 \%\right)$ and AUC was 0.956 (95\% CI 0.927-0.987) (Table 2, Fig. 2C). For the third sensitivity analysis based on TC, DOR was 189 (95\% CI 106-337, $\left.\mathrm{I}^{2}=27.2 \%\right)$ and AUC was 0.979 (95\% CI 0.970-0.988) (Table 2, Fig. 2D).

According to the 42 cohorts, the summary estimate of sensitivity was 0.911 (95\% CI $0.871-0.940)$ and the summary estimate of specificity was 0.912 (95\% CI $0.892-0.928)$. These sensitivity and specificity estimates yielded PLR of 10.4 (95\% CI 8.4-12.7) and NLR of 0.098 (95\% CI 0.066-0.142). Based on Grimes' criteria, these likelihood ratios suggested a large increase and decrease of probabilities, respectively ${ }^{27}$. PLR and NLR calculated in subgroup analysis focusing on non-high-risk cohorts and TC reference also suggested large increase and decrease of probabilities, respectively. However, PLR and NLR calculated in sensitivity analysis focusing on CCNA reference suggested a moderate increase and decrease of probabilities, respectively. 


\begin{tabular}{|c|c|c|c|c|c|c|c|c|c|}
\hline Cohort name & Country & \begin{tabular}{|l|} 
Study \\
design
\end{tabular} & \begin{tabular}{|l|} 
Report \\
type
\end{tabular} & Specimen type & Facility & $\begin{array}{l}\text { Reference } \\
\text { test }\end{array}$ & Index test & Comparisons & \begin{tabular}{|l|}
$\begin{array}{l}\text { Non- } \\
\text { high-risk }\end{array}$ \\
\end{tabular} \\
\hline Alfa (2002) & Canada & pCohort & Full A & s/o CDI & A tertiary hospital & CCNA & Triage & 400 & Yes \\
\hline Anonymous (2011) Ind:Pre & & pCohort & C Abst & Liquid stool & & TC & Premiere & 260 & Yes \\
\hline Anonymous (2011) Ind:QC & & pCohort & C Abst & Liquid stool & & TC & Quik Chek & 260 & Yes \\
\hline Barbut (2000) & France & Cohort & Full A & Diarrhea & Hospitals & CCNA & Triage & 304 & Yes \\
\hline Barkin (2012) & USA & rCohort & Full A & s/o CDI & A community teaching hospital & TC & ImmunoCard & 267 & Yes \\
\hline Bennett (1989) & USA & Cohort & Full A & Diarrhea & Nursing homes & CCNA & Culturette & 142 & Yes \\
\hline Broeck (2010) & Belgium & Cohort & C Post & Diarrhea & A university hospital & TC & Quik Chek & 265 & Yes \\
\hline Broukhanski (2011) & Canada & & C Abst & & & TC & Chek-60 & 265 & Yes \\
\hline Brown (2011) & USA & Cohort & Full A & Liquid stool & A test center & CCNA & Chek-60 & 357 & Yes \\
\hline Bruins (2012) & Netherlands & Cohort & Full A & s/o CDI & A laboratory & TC & Quik Chek & 986 & Yes \\
\hline Clark (2011) & UK & pCohort & Letter & Diarrhea & A hospital & CCNA & Chek-60 & 322 & Yes \\
\hline Devlin (2011) & Canada & Cohort & C Abst & & & TC & Chek-60 & 200 & Yes \\
\hline Eastwood (2009) Ref:CCNA & UK & pCohort & Full A & Diarrhea & Teaching hospitals & CCNA & Chek-60 & 558 & Yes \\
\hline Eastwood (2009) Ref:TC & UK & pCohort & Full A & Diarrhea & Teaching hospitals & TC & Chek-60 & 564 & Yes \\
\hline Eckert (2014) Ref:CCNA & France & pCohort & Full A & s/o CDI, diarrhea & A test center & CCNA & Quik Chek & 308 & Yes \\
\hline Eckert (2014) Ref:TC & France & pCohort & Full A & s/o CDI, diarrhea & A test center & TC & Quik Chek & 308 & Yes \\
\hline Fille (1998) & Austria & Cohort & Full A & s/o CDI & A laboratory & CCNA & Culturette & 103 & No \\
\hline Goldenberg (2010) & UK & Cohort & Full A & Diarrhea & A hospital & TC & Chek & 500 & Yes \\
\hline Greene (2010) & USA & & C Abst & & & CCNA & Chek-60 & 236 & No \\
\hline Jacobs (1996) & Israel & Cohort & Full A & Diarrhea & A teaching hospital & TC & Culturette & 259 & Yes \\
\hline Johnstone (2010) & Canada & & C Abst & & & TC & Chek-60 & 180 & Yes \\
\hline Jung (1990) & Sweden & Cohort & Full A & s/o CDI & & CCNA & Culturette & 380 & Yes \\
\hline Kawada (2011) & Japan & pCohort & Full A & s/o CDI & A hospital & TC & Quik Chek & 60 & Yes \\
\hline $\operatorname{Kim}(2014)$ & Korea & Cohort & Full A & s/o CDI, loose stool & A tertiary teaching hospital & TC & Quik Chek & 599 & Yes \\
\hline Kvach (2010) & USA & Cohort & Full A & Liquid stool/Diarrhea & A hospital & CCNA & Chek-60 & 400 & Yes \\
\hline Landry (2001) & USA & pCohort & Full A & & A hospital & CCNA & Triage & 90 & No \\
\hline Larson (2010) & USA & Cohort & Full A & Soft/liquid stool & A medical center & CCNA & Quik Chek & 699 & Yes \\
\hline Massey (2003) & Canada & Cohort & Full A & s/o CDI & & CCNA & Triage & 557 & Yes \\
\hline Miller (2013) Ind:C60 & USA & Cohort & Full A & s/o CDI, liquid stool & A university hospital & CCNA & Chek-60 & 381 & Yes \\
\hline Miller (2013) Ind:QC & USA & Cohort & Full A & s/o CDI, liquid stool & A university hospital & CCNA & Quik Chek & 381 & Yes \\
\hline Ota (2012) & USA & pCohort & Full A & Liquid stool & A children hospital & CCNA & Quik Chek & 141 & Yes \\
\hline Peterson (2011) Ind:C60 & USA & Cohort & Full A & s/o CDI & university laboratory & TC & Chek-60 & 1000 & Yes \\
\hline Peterson (2011) Ind:QC & USA & Cohort & Full A & s/o CDI & university laboratory & TC & Quik Chek & 1000 & Yes \\
\hline Planche (2013) Ref:CCNA & UK & pCohort & Full A & Bristol 5-7 & Teaching hospitals & CCNA & Chek-60 & 12329 & Yes \\
\hline Planche (2013) Ref:TC & UK & pCohort & Full A & Bristol 5-7 & Teaching hospitals & TC & Chek-60 & 12365 & Yes \\
\hline Qutab (2011) & Saudi Arabia & Cohort & Full A & s/o CDI & & CCNA & Chek- 60 & 150 & Yes \\
\hline Reller (2010) Ind:C60 & USA & Cohort & Full A & & & CCNA & Chek-60 & 600 & Yes \\
\hline Reller (2010) Ind:QC & USA & Cohort & Full A & & & CCNA & Quik Chek & 600 & Yes \\
\hline Selvaraju (2011) & USA & Cohort & Full A & Liquid/soft/formed stool & & TC & Quik Chek & 200 & No \\
\hline Shah (2010) & USA & Cohort & C Abst & & & TC & Chek-60 & 401 & Yes \\
\hline Staneck (1996) Ind:Cul & USA & rCohort & Full A & AAD & University hospitals & CCNA & Culturette & 901 & No \\
\hline Staneck (1996) Ind:IC & USA & rCohort & Full A & AAD & University hospitals & CCNA & ImmunoCard & 906 & No \\
\hline Swindells (2010) Ref:CCNA & UK & Cohort & Full A & Diarrhea, $>65 y o$ & & CCNA & Quik Chek & 150 & Yes \\
\hline Swindells (2010) Ref:TC & UK & Cohort & Full A & Diarrhea, $>66$ yo & & TC & Quik Chek & 150 & Yes \\
\hline Ticehurst (2006) & USA & Cohort & Full A & & Teaching hospitals & CCNA & Chek-60 & 366 & Yes \\
\hline Turan (2011) Ref:CCNA & Turkey & Cohort & C Abst & s/o CDI & & CCNA & Quik Chek & 132 & Yes \\
\hline Turan (2011) Ref:TC & Turkey & Cohort & C Abst & s/o CDI & & TC & Quik Chek & 132 & Yes \\
\hline Turgeon (2003) Ind:IC & USA & Cohort & Full A & Stool with any consistency & Hosptals & CCNA & ImmunoCard & 1003 & No \\
\hline Turgeon (2003) Ind:Tri & USA & Cohort & Full A & Stool with any consistency & Multicenter & CCNA & Triage & 1002 & No \\
\hline Vanpoucke (2001) & Belgium & Cohort & Full A & $\begin{array}{l}\text { s/o CDI, liquid/semi- } \\
\text { liquid stool }\end{array}$ & A university hospital & CCNA & Culturette & 366 & Yes \\
\hline Walkty (2013) & Canada & Cohort & Full A & Diarrhea & A hospital and laboratories & TC & Quik Chek & 428 & Yes \\
\hline Wren (2009) & UK & Cohort & Full A & s/o CDI & & TC & & 1007 & Yes \\
\hline Zheng (2004) Ind:C30 & USA & Cohort & Full A & & Test centers & CCNA & \begin{tabular}{|l|} 
Chek-30 \\
\end{tabular} & 992 & Yes \\
\hline Zheng (2004) Ind:C60 & USA & Cohort & Full A & & Test centers & CCNA & Chek-60 & 992 & Yes \\
\hline
\end{tabular}

Table 1. Characteristics of included cohorts. When a report compared an index test with two reference tests or when a report compared two index test with a reference, we regarded such a report as two independent study. <Cohort name $>$ Ref: Reference test. Ind: Index test. Pre: Premiere. QC: Quik Chek. C60: Chek-60. IC: ImmunoCard. Cul: Culturette. Tri: Triage. C30: Chek-30. <Study design > pCohort: prospective cohort. rCohort: retrospective cohort. $<$ Report type $>$ Full A: full-length article. C Abst: conference abstract.

C Post: conference poster. $<$ Specimen type $>$ s/o CDI: suspected of C. difficiele. AAD: Antibiotics-associated diarrhea $<$ Reference test $>$ CCNA: Cell cytotoxicity neutralization assay. TC: Toxigenic culture. 
Chek-60

Study

Broukhanski (2011)

Brown (2011)

Clark (2011)

Devlin (2011)

Eastwood (2009) Ref:CCNA

Eastwood (2009) Ref:TC

Greene (2010)

Johnstone (2010)

Kvach (2010)

Miller (2013) Ind:C60

Peterson (2011) Ind:C60

Planche (2013) Ref:CCNA

Planche (2013) Ref:TC

Qutab (2011)

Reller (2010) Ind:C60

Shah (2010)

Ticehurst (2006)

Zheng (2004) Ind:C60

Quik Chek

Study

Anonymous (2011) Ind:QC

Broeck (2010)

Bruins (2012)

Eckert (2014) Ref:CCNA

Eckert (2014) Ref:TC

Kawada (2011)

Kim (2014)

Larson (2010)

Miller (2013) Ind: QC

Ota (2012)

Peterson (2011) Ind:QC

Reller (2010) Ind:QC

Selvaraju (2011)

Swindells (2010) Ref:CCNA

Swindells (2010) Ref:TC

Turan (2011) Ref:CCNA

Turan (2011) Ref:TC

Walkty (2013)

Culturette

Study

Bennett (1989)

Fille (1998)

Jacobs (1996)

Jung (1990)

Staneck (1996) Ind:Cul

Vanpoucke (2001)

Triage

Study

Alfa (2002)

Barbut (2000)

Landry (2001)

Massey (2003)

Turgeon (2003) Ind:Tri

ImmunoCard

Study

Barkin (2012)

Staneck (1996) Ind:IC

Turgeon (2003) Ind:IC

Chek-30

Study

Zheng (2004) Ind:C30

Premiere

Study

Anonymous (2011) Ind:Pre

Not known

Study

Goldenberg (2010)

Wren (2009)

$\begin{array}{rrrrc}\text { TP } & \text { FP } & \text { FN } & \text { TN } & \text { Sensitivity }(95 \% \text { Cl) } \\ 111 & 12 & 2 & 140 & 0.98[0.94,1.00] \\ 35 & 22 & 0 & 300 & 1.00[0.90,1.00] \\ 14 & 30 & 0 & 278 & 1.00[0.77,1.00] \\ 24 & 5 & 0 & 171 & 1.00[0.86,1.00] \\ 82 & 33 & 9 & 434 & 0.90[0.82,0.95] \\ 92 & 26 & 13 & 433 & 0.88[0.80,0.93] \\ 10 & 28 & 0 & 198 & 1.00[0.69,1.00] \\ 15 & 11 & 1 & 153 & 0.94[0.70,1.00] \\ 88 & 112 & 0 & 200 & 1.00[0.96,1.00] \\ 85 & 77 & 2 & 217 & 0.98[0.92,1.00] \\ 122 & 55 & 24 & 799 & 0.84[0.77,0.89] \\ 705 & 902 & 26 & 10696 & 0.96[0.95,0.98] \\ 977 & 624 & 57 & 10707 & 0.94[0.93,0.96] \\ 49 & 12 & 3 & 86 & 0.94[0.84,0.99] \\ 42 & 55 & 4 & 499 & 0.91[0.79,0.98] \\ 66 & 13 & 6 & 316 & 0.92[0.83,0.97] \\ 39 & 35 & 1 & 291 & 0.97[0.87,1.00] \\ 127 & 93 & 10 & 762 & 0.93[0.87,0.96]\end{array}$

pecificity $(95 \% \mathrm{Cl}) \quad$ Sensitivity $(95 \% \mathrm{Cl}) \quad$ Specificity $(95 \% \mathrm{Cl})$

$0.92[0.87,0.96]$

$0.93[0.90,0.96]$

$0.90[0.86,0.93]$

$0.97[0.93,0.99]$

$0.93[0.90,0.95]$

$0.94[0.92,0.96]$

$0.88[0.83,0.92]$

$0.93[0.88,0.97]$

$0.64[0.59,0.69]$

$0.74[0.68,0.79]$

$0.94[0.92,0.95]$

$0.92[0.92,0.93]$

$0.94[0.94,0.95]$

$0.88[0.80,0.94]$

$0.90[0.87,0.92]$

$0.96[0.93,0.98]$

$0.89[0.85,0.92]$

$0.89[0.87,0.91]$

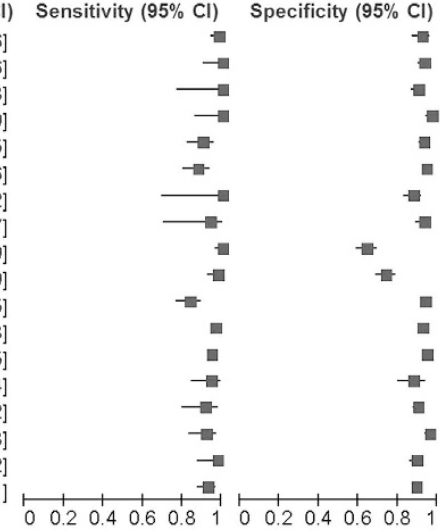

TP $F P$ FN TN

$\begin{array}{llll}18 & 1 & 1 & 240\end{array}$

$\begin{array}{llll}48 & 14 & 6 & 197\end{array}$

$\begin{array}{llll}71 & 22 & 2 & 891\end{array}$

$\begin{array}{llll}23 & 25 & 0 & 260\end{array}$

$\begin{array}{llll}35 & 13 & 1 & 259\end{array}$

$\begin{array}{llll}28 & 4 & 0 & 28\end{array}$

$\begin{array}{llll}91 & 38 & 9 & 461\end{array}$

$\begin{array}{llll}47 & 67 & 0 & 585\end{array}$

$\begin{array}{llll}86 & 69 & 1 & 225\end{array}$

$\begin{array}{llll}21 & 21 & 5 & 94\end{array}$

$\begin{array}{llll}128 & 47 & 18 & 807\end{array}$

$\begin{array}{llll}46 & 94 & 0 & 460\end{array}$

$\begin{array}{llll}47 & 27 & 1 & 125\end{array}$

$\begin{array}{llll}15 & 7 & 0 & 128\end{array}$

$\begin{array}{llll}18 & 4 & 0 & 128\end{array}$

$\begin{array}{llll}10 & 8 & 14 & 100\end{array}$

$\begin{array}{llll}11 & 7 & 10 & 104\end{array}$

$\begin{array}{llll}52 & 11 & 11 & 354\end{array}$

TP FP FN TN

$\begin{array}{llll}12 & 12 & 1 & 117\end{array}$

$\begin{array}{llll}11 & 5 & 10 & 77\end{array}$

$\begin{array}{llll}9 & 35 & 10 & 205\end{array}$

$\begin{array}{llll}71 & 59 & 15 & 235\end{array}$

$\begin{array}{llll}86 & 39 & 42 & 734\end{array}$

$\begin{array}{llll}13 & 3 & 43 & 307\end{array}$

TP FP FN TN

$\begin{array}{llll}53 & 38 & 5 & 304\end{array}$

$\begin{array}{llll}33 & 39 & 1 & 231\end{array}$

$\begin{array}{llll}15 & 13 & 1 & 61\end{array}$

$\begin{array}{llll}139 & 57 & 4 & 357\end{array}$

$\begin{array}{llll}90 & 93 & 11 & 808\end{array}$

TP FP FN TN

$\begin{array}{llll}35 & 0 & 0 & 232\end{array}$

$\begin{array}{llll}108 & 63 & 20 & 715\end{array}$

$\begin{array}{llll}81 & 68 & 20 & 834\end{array}$

TP FP FN TN

$\begin{array}{llll}129 & 89 & 8 & 766\end{array}$

TP FP FN TN

$\begin{array}{llll}18 & 2 & 1 & 239\end{array}$

Sensitivity $(95 \% \mathrm{Cl})$
$0.95[0.74,1.00]$
$0.89[0.77,0.96]$
$0.97[0.90,1.00]$
$1.00[0.85,1.00]$
$0.97[0.85,1.00]$
$1.00[0.88,1.00]$
$0.91[0.84,0.96]$
$1.00[0.92,1.00]$
$0.99[0.94,1.00]$
$0.81[0.61,0.93]$
$0.88[0.81,0.93]$
$1.00[0.92,1.00]$
$0.98[0.89,1.00]$
$1.00[0.78,1.00]$
$1.00[0.81,1.00]$
$0.42[0.22,0.63]$
$0.52[0.30,0.74]$
$0.83[0.71,0.91]$

$0.94[0.89,0.97]$

$1.00[0.98,1.00]$

$0.93[0.89,0.96]$

$0.98[0.96,0.98]$

$0.91[0.87,0.94]$

$0.95[0.92,0.97]$

$0.88[0.71,0.96]$

$0.92[0.90,0.95]$

$0.90[0.87,0.92]$

$0.77[0.71,0.81]$

$0.82[0.73,0.88]$

$0.94[0.93,0.96]$

$0.83[0.80,0.86]$

$0.82[0.75,0.88]$

$0.95[0.90,0.98]$

$0.97[0.92,0.99]$

$0.93[0.86,0.97]$

$0.94[0.87,0.97$

$0.97[0.95,0.98]$

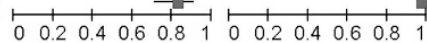

ecificity $(95 \% \mathrm{Cl})$ Sensitivity $(95 \% \mathrm{Cl}) \quad$ Specificity $(95 \% \mathrm{Cl})$

$0.91[0.84,0.95]$

$0.94[0.86,0.98]$

$0.85[0.80,0.90]$

$0.80[0.75,0.84]$

$0.95[0.93,0.96]$

$0.99[0.97,1.00]$

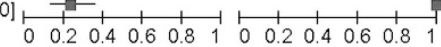

ensitivity $(95 \% \mathrm{Cl})$ Specificity $(95 \% \mathrm{Cl})$ Sensitivity $(95 \% \mathrm{Cl}) \quad$ Specificity $(95 \% \mathrm{Cl})$ $0.89[0.85,0.92]$ $0.86[0.81,0.90]$

$0.82[0.72,0.90]$

$0.86[0.83,0.89]$

$0.90[0.88,0.92]$

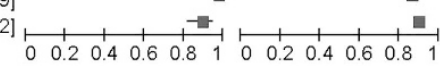

ensitivity $(95 \% \mathrm{Cl})$ Specificity $(95 \% \mathrm{Cl})$ Sensitivity $(95 \% \mathrm{Cl}) \quad$ Specificity $(95 \% \mathrm{Cl})$

$1.00[0.98,1.00]$

$0.92[0.90,0.94]$

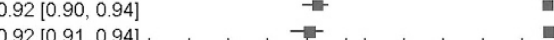

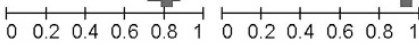

ificity $(95 \% \mathrm{Cl})$ Sensitivity $(95 \% \mathrm{Cl}) \quad$ Specificity $(95 \% \mathrm{Cl})$ $0.90[0.87,0.92]$

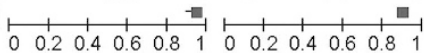

ensitivity $(95 \% \mathrm{Cl})$ Specificity $(95 \% \mathrm{Cl})$ Sensitivity $(95 \% \mathrm{Cl}) \quad$ Specificity $(95 \% \mathrm{Cl})$ $0.95[0.74,1.00]$ $0.99[0.97,1.00]$

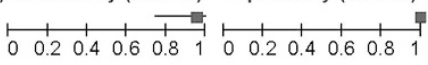

TP FP FN TN

$\begin{array}{lllll}36 & 64 & 0 & 400\end{array}$

$\begin{array}{llll}83 & 40 & 4 & 880\end{array}$
Sensitivity $(95 \% \mathrm{Cl})$ Specificity $(95 \% \mathrm{Cl})$ Sensitivity $(95 \% \mathrm{Cl}) \quad$ Specificity $(95 \% \mathrm{Cl})$ $1.00[0.90,1.00]$ $0.95[0.89,0.99]$

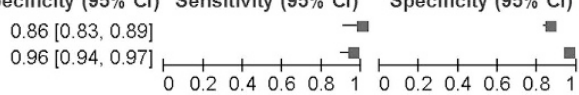

Figure 1. Paired forest plot. TP: true positive. FP: false positive. FN: false negative. TN: true negative. Ind: index test. Pre: Premiere. QC: Quik Chek. C60: Chek 60. Cul: Culturette. Tri: Triage. Ref: reference text. CCNA: cell cytotoxicity neutralization assay. TC: toxigenic culture. 


\begin{tabular}{|c|c|c|c|c|c|c|c|c|}
\hline & (A) & (B) & (C) & (D) & (E) & (F) & (G) & (H) \\
\hline Reference & Any & Any & CCNA & TC & Any & Any & Any & Any \\
\hline Index & Any & Any & Any & Any & Chek-60 & Quik Chek & Culturette & Triage \\
\hline Non-high-risk & Any & Yes & Any & Any & Any & Any & Any & Any \\
\hline $\mathrm{N}$ & 42 & 6 & 26 & 21 & 16 & 15 & 6 & 5 \\
\hline $\mathrm{n}$ & 29243 & 2745 & 22366 & 20396 & 18737 & 6209 & 2151 & 2353 \\
\hline DOR & 115 & 189 & 80 & 189 & 159 & 152 & 22 & 97 \\
\hline$(95 \% \mathrm{CI})$ & $(77-172)$ & $(54-660)$ & $(50-131)$ & $(106-337)$ & $(104-243)$ & $(75-308)$ & $(11-43)$ & $(61-154)$ \\
\hline $\mathrm{I}^{2}$ & $12.0 \%$ & $0 \%$ & $0 \%$ & $27.2 \%$ & $0 \%$ & $13.0 \%$ & $9.5 \%$ & $0 \%$ \\
\hline AUC & 0.970 & 0.986 & 0.956 & 0.979 & 0.979 & 0.980 & 0.852 & 0.975 \\
\hline$(95 \% \mathrm{CI})$ & $(0.958-0.982)$ & \begin{tabular}{|l}
$(0.976-0.998)$ \\
\end{tabular} & \begin{tabular}{|l}
$(0.927-0.987)$ \\
\end{tabular} & \begin{tabular}{|l}
$(0.970-0.988)$ \\
\end{tabular} & $(0.970-0.989)$ & \begin{tabular}{|l}
$(0.968-0.992)$ \\
\end{tabular} & \begin{tabular}{|l}
$(0.794-0.918)$ \\
\end{tabular} & \begin{tabular}{|l}
$(0.959-0.991)$ \\
\end{tabular} \\
\hline Sensitivity & 0.911 & 0.919 & 0.901 & 0.914 & 0.942 & 0.925 & 0.610 & 0.943 \\
\hline$(95 \% \mathrm{CI})$ & $(0.871-0.940)$ & $(0.861-0.955)$ & $(0.838-0.941)$ & $(0.865-0.947)$ & \begin{tabular}{|l}
$(0.913-0.962)$ \\
\end{tabular} & $(0.857-0.962)$ & $(0.600-0.786)$ & $(0.891-0.971)$ \\
\hline Specificity & 0.912 & 0.929 & 0.894 & 0.941 & 0.901 & 0.918 & 0.929 & 0.874 \\
\hline$(95 \% \mathrm{CI})$ & $(0.892-0.928)$ & \begin{tabular}{|l}
$(0.867-0.964)$ \\
\end{tabular} & $(0.867-0.916)$ & $(0.922-0.955)$ & $(0.867-0.927)$ & $(0.879-0.945)$ & \begin{tabular}{|l}
$(0.843-0.969)$ \\
\end{tabular} & $(0.851-0.895)$ \\
\hline PLR & 10.4 & 12.9 & 8.5 & 15.5 & 9.5 & 11.3 & 8.6 & 7.5 \\
\hline$(95 \% \mathrm{CI})$ & $(8.4-12.7)$ & $(6.8-25.2)$ & $(6.7-10.8)$ & $(11.7-20.4)$ & $(7.1-12.9)$ & $(7.6-16.8)$ & $(3.8-20.1)$ & $(6.2-8.9)$ \\
\hline NLR & 0.098 & 0.087 & 0.111 & 0.091 & 0.064 & 0.082 & 0.420 & 0.065 \\
\hline$(95 \% \mathrm{CI})$ & $(0.066-0.142)$ & \begin{tabular}{|l}
$(0.049-0.152)$ \\
\end{tabular} & \begin{tabular}{|l}
$(0.066-0.181)$ \\
\end{tabular} & $(0.057-0.144)$ & $(0.042-0.097)$ & $(0.041-0.156)$ & $(0.312-0.548)$ & $(0.033-0.125)$ \\
\hline
\end{tabular}

Table 2. Summary of results N: number of cohorts. n: number of comparisons. SROC: summary receiver operating characteristics. AUC: area under hierarchical summary receiver operating characteristics curve. PLR: positive likelihood ratio. NLR: negative likelihood ratio. 95\% CI: 95\% confidence interval.

\section{(A) All studies}

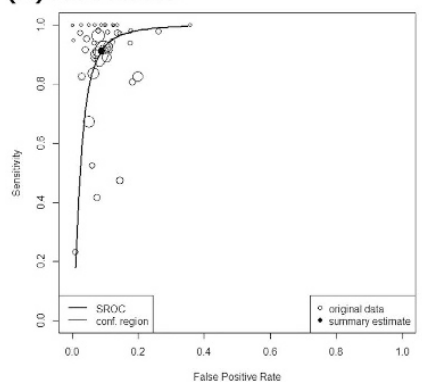

(D) Toxigenic culture

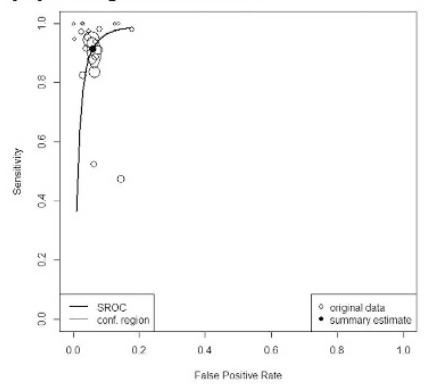

(G) Culturette

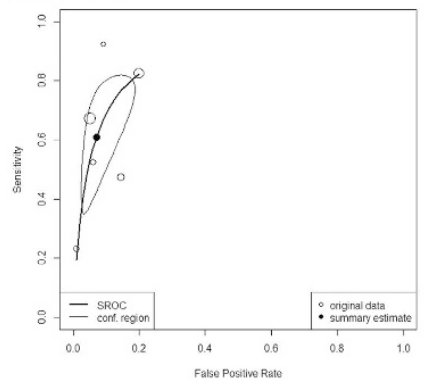

(B) Non-high risk studies

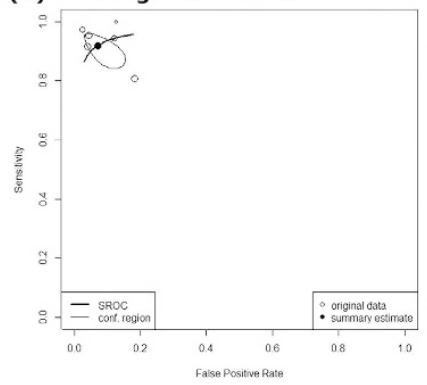

(E) Check-60

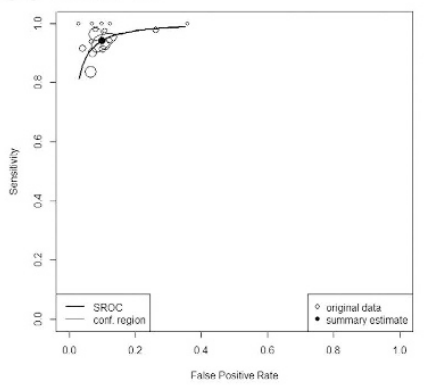

(H) Triage

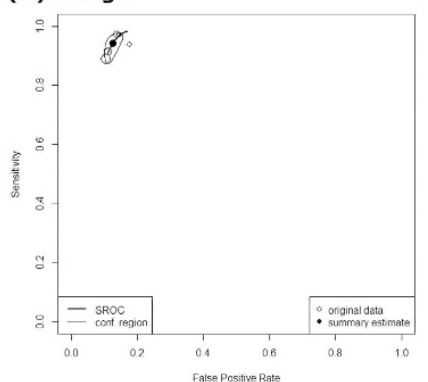

(C) Cell cytotoxicity neutralization assay

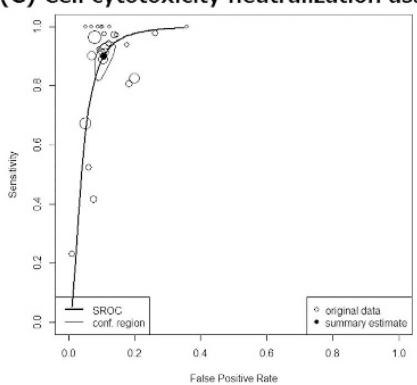

(F) Quik Chek

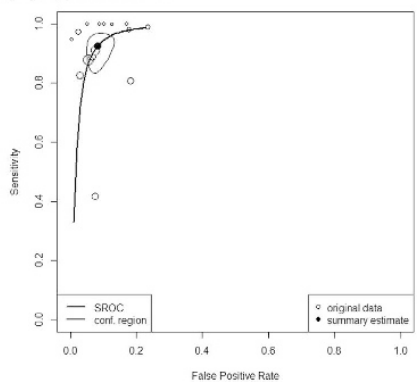

Figure 2. Hierarchical summary receiver-operator characteristic curves. 


\begin{tabular}{|c|c|c|c|c|c|c|c|c|c|c|}
\hline \multirow[b]{2}{*}{ Outcome } & \multirow[b]{2}{*}{$\begin{array}{l}\mathrm{N} \text { of studies/ } \\
\text { patients }\end{array}$} & \multirow[b]{2}{*}{ Study design } & \multicolumn{5}{|c|}{ Factors that may decrease quality of evidence } & \multicolumn{2}{|c|}{ Effect per 1000 patients tested } & \multirow[b]{2}{*}{$\begin{array}{c}\text { Test accuracy } \\
\text { quality of } \\
\text { evidence }\end{array}$} \\
\hline & & & $\begin{array}{c}\text { Risk of } \\
\text { bias }\end{array}$ & Indirectness & Inconsistency & Imprecision & $\begin{array}{c}\text { Publication } \\
\text { bias }\end{array}$ & $\begin{array}{c}\text { PTP of } 15 \% \\
\text { (low estimation) }\end{array}$ & $\begin{array}{l}\text { PTP of } 25 \% \\
\text { (high } \\
\text { estimation) }\end{array}$ & \\
\hline True positives & 42 groups & \multirow{2}{*}{$\begin{array}{c}\text { cohort \& } \\
\text { case-control }\end{array}$} & \multirow{2}{*}{ serious $^{\#}$} & \multirow{2}{*}{ not serious } & \multirow{2}{*}{ not serious } & \multirow{2}{*}{ not serious } & \multirow{2}{*}{ none } & $137(131$ to 141$)$ & $228(218$ to 235$)$ & \multirow{2}{*}{$\underset{\text { Moderate }}{\bigoplus \bigoplus \bigoplus}$} \\
\hline False negatives & 3055 subjects & & & & & & & $13(9$ to 19$)$ & $22(15$ to 32$)$ & \\
\hline True negatives & 42 groups & \multirow{2}{*}{$\begin{array}{c}\text { cohort \& } \\
\text { case-control }\end{array}$} & \multirow{2}{*}{ serious $\#$} & \multirow{2}{*}{ not serious } & \multirow{2}{*}{ not serious } & \multirow{2}{*}{ not serious } & \multirow{2}{*}{ none } & 775 (758 to 789$)$ & $684(669$ to 696$)$ & \multirow{2}{*}{$\underset{\text { Moderate }}{\bigoplus \bigoplus \bigoplus}$} \\
\hline False positives & 26188 subjects & & & & & & & 75 (61 to 92$)$ & $66(54$ to 81$)$ & \\
\hline
\end{tabular}

Table 3. GRADE evidence profile for diagnostic test accuracy by detecting glutamate dehydrogenase assay for Clostridium difficile infection (CDI). This table was based on following statistics: sensitivity 0.911 (95\% CI: 0.871 to 0.940 ), specificity 0.912 ( $95 \%$ CI: 0.892 to 0.928$)$, prevalence $15-25 \%$. PTP: pre-test probability. "Most studies had high risk for "flow and timing".

GRADE Evidence Profile was presented as Table 3. Supposing the protest pretest probability is in the range $15-25 \%^{2}$, among 1000 tested subjects, there are 137-228 true positives, $12-22$ false negatives, 684-775 true negatives, and 66-75 false positives. PPV was $65-78 \%$ and NPV was $97-98 \%$.

Subgroup analysis based on index test. Check-60 was evaluated in 16 cohorts with 18737 comparisons. The DOR of 159 and AUC of 0.979 suggested excellent overall diagnostic accuracy. The sensitivity was 0.942 and the specificity was 0.901 . The PLR of 9.5 and NLR of 0.064 suggested moderate increase and large decrease of likelihood ratio, respectively (Table 2, Figure 2E).

Quik Chek was evaluated in 15 cohorts with 6205 comparisons. The DOR of 152 and AUC of 0.980 also suggested excellent overall diagnostic accuracy. The sensitivity was 0.925 and the specificity was 0.918 . The PLR of 11.3 and NLR of 0.082 suggested a large increase/decrease of the likelihood ratio (Table 2, Figure 2F).

Six cohorts evaluated the Culturette Latex agglutination test with 2151 comparisons. The AUC was 0.852 (95\% CI 0.794-0.918) suggesting good overall diagnostic accuracy. The summary estimate of sensitivity of 0.610 was lower than those by Chek-60 and Quik Chek. The PLR was 8.6, which suggested a moderate increase of probability when the test is positive. The NLR was 0.420 , which meant a small decrease of probability when the test is negative (Table 2, Figure $2 \mathrm{G}$ ).

Five cohorts with 2353 comparisons assessed the diagnostic accuracy of Triage. Though excellent overall diagnostic accuracy was revealed by the AUC of 0.975 , the specificity and PLR were lower than those for the other three assay kits (Table 2, Figure $2 \mathrm{H}$ ).

\section{Discussion}

To the best of our knowledge, this is the first meta-analysis to provide the summary estimate sensitivity and specificity of GDH detection for CDI. Our analysis showed that detecting GDH had excellent AUC and that test results from GDH greatly changed the probability of CDI. We believe that our result was robust for the careful study search, the use of hierarchical model, and low heterogeneity indicated by $\mathrm{I}^{2}<30 \%$. The quality-based subgroup analysis that replicated the results from all studies with any quality also support the robustness.

Reference-test-based sensitivity analysis revealed slightly discrepant results. When GDH assay was compared to reference test TC, the overall test accuracy was excellent. However, GDH assay seemed to have lower specificity when compared to reference test CCNA. Though both CCNA and TC are regarded as established standard examination for CDI, these two tests sometimes exhibit conflicting results. A large-scale prospective study by Planche et al. suggested that CCNA is a better reference test compared to TC because CCNA more accurately reflect mortality and $\mathrm{CDI}^{23}$. If we trust only the CCNA reference, the diagnostic accuracy of the GDH assay seems slightly degraded (Table 2, Figure 2C).

Index based subgroup analyses revealed that Chek-60 and Quik Chek, which were the most frequently evaluated kits, had the best performance. Although not supported by a sufficient number of studies, Triage seemed to lack specificity. The Culturette Brand Rapid Latex Test for CDI had clearly low diagnostic performance. Even though it detected GDH, this test was not designed for GDH. We have currently no reason to use the Culturette Brand Latex Test to detect GDH.

Once we assume the pretest probability was in the range $15-25 \%$, PPV was $65-78 \%$ and NPV was $97-98 \%$. While the GDH assay negative result is generally trustful, a positive GDH assay leads to wrong diagnosis for a third or a fourth of the tested population. Therefore, the currently used multi-step algorithm is a reasonable solution. In the medical resource abundant situation, NAATs can provide quick and accurate results for the second step. If use of NAATs is restricted, toxin detection is an alternative. However, toxin detection is not sensitive enough. Thus, we have to apply the NAATs as third step for GDH-positive toxin-negative specimens ${ }^{31}$. Even though some epidemiologic studies have suggested that CDI accounts for 15-25\% of antibiotics-associated diarrhea, pretest probability should be judged by clinicians considering the patient's clinical background and epidemiology in the area. Thus, the result of a GDH assay can be carefully interpreted.

To diagnose CDI in clinical practice, biochemical examinations that detect GDH, as well as toxin or nucleic acied of C. difficile in the stool of CDI-suspected patients are widely used. GDH is a metabolic enzyme that converts glutamate to $\alpha$-ketoglutarate ${ }^{8-11}$. This enzyme commonly presents in many eukaryotes and microbes including C. difficile and other Clostridium species. To detect GDH in the stool, latex agglutination test was formerly used, whereas quantitative immunoassays are common these days. The key advantage of the enzyme immunoassays over the latex agglutination test is enhanced sensitivity due to quantitative evaluation using a standard 
curve. Moreover, the recently available lateral flow assay does not require a trained technician. Nowadays, we can obtain simple and accurate commercially-available enzyme immunoassay kits at low price though CCNA and TC are regarded as standard.

We need to comment on the limitations of our study. First, some of the included studies had high risk or high applicability concerns, therefore, we need to conduct sensitivity analysis excluding these studies. Second, subgroup analysis concerning the Culturette Latex test and Triage included a small number of studies; thus results were not sufficiently trustful. Third, the results were not consistent according to the reference tests. Thus, we provided GDH assay accuracies using two references separately. We believe these data are useful for future research. Fourth, recent advancement of PCR technique enables detection of a scarce load of microbes. PCR may be able to detect C. diff with higher sensitivity than culture though the culture is usually regarded as the gold standard. If we had used PCR as reference standard, the specificity would have been improved ${ }^{32}$.

In conclusion, we performed a systematic review and meta-analysis of the diagnostic test accuracy of detecting GDH for the diagnosis of CDI using a hierarchical model and a sufficient number of studies and comparisons. According to our analysis using 42 cohorts consisting of 29243 comparisons, the overall test accuracy was excellent, sensitivity was 0.911 , specificity was 0.912 , and the positive/negative results largely increased/decreased the probability of CDI. Suppose pretest probability was 15-25\%, PPV was 65-78\% and NPV was 97-98\%.

\section{References}

1. Kelly, C. P. \& LaMont, J. T. Clostridium difficile-more difficult than ever. N Engl J Med 359, 1932-1940 (2008).

2. Bartlett, J. G. Antibiotic-associated diarrhea. Clin Infect Dis 15, 573-581 (1992).

3. Janarthanan, S., Ditah, I., Adler, D. G. \& Ehrinpreis, M. N. Clostridium difficile-associated diarrhea and proton pump inhibitor therapy: a meta-analysis. Am J Gastroenterol 107, 1001-1010 (2012).

4. Kwok, C. S. et al. Risk of Clostridium difficile infection with acid suppressing drugs and antibiotics: meta-analysis. Am J Gastroenterol 107, 1011-1019 (2012).

5. van Nood, E. et al. Duodenal infusion of donor feces for recurrent Clostridium difficile. N Engl J Med 368, 407-415 (2013).

6. Lagier, J. C. et al. Dramatic reduction in Clostridium difficile ribotype 027-associated mortality with early fecal transplantation by the nasogastric route: a preliminary report. Eur J Clin Microbiol Infect Dis 34, 1597-1601 (2015).

7. Gravel, D. et al. Health care-associated Clostridium difficile infection in adults admitted to acute care hospitals in Canada: a Canadian Nosocomial Infection Surveillance Program Study. Clin Infect Dis 48, 568-576 (2009).

8. Surawicz, C. M. et al. Guidelines for diagnosis, treatment, and prevention of Clostridium difficile infections. Am J Gastroenterol 108, 478-498; quiz 499 (2013).

9. Bagdasarian, N., Rao, K. \& Malani, P. N. Diagnosis and treatment of Clostridium difficile in adults: a systematic review. JAMA 313, 398-408 (2015).

10. Planche, T. et al. Diagnosis of Clostridium difficile infection by toxin detection kits: a systematic review. Lancet Infect Dis 8, 777-784 (2008).

11. Crobach, M. J., Dekkers, O. M., Wilcox, M. H. \& Kuijper, E. J. European Society of Clinical Microbiology and Infectious Diseases (ESCMID): data review and recommendations for diagnosing Clostridium difficile-infection (CDI). Clin Microbiol Infect 15, 1053-1066 (2009).

12. Deshpande, A. et al. Diagnostic accuracy of real-time polymerase chain reaction in detection of Clostridium difficile in the stool samples of patients with suspected Clostridium difficile Infection: a meta-analysis. Clin Infect Dis 53, e81-90 (2011).

13. Wei, C., Wen-En, L., Yang-Ming, L., Shan, L. \& Yi-Ming, Z. Diagnostic accuracy of loop-mediated isothermal amplification in detection of Clostridium difficile in stool samples: a meta-analysis. Arch Med Sci 11, 927-936 (2015).

14. O’Horo, J. C., Jones, A., Sternke, M., Harper, C. \& Safdar, N. Molecular techniques for diagnosis of Clostridium difficile infection: systematic review and meta-analysis. Mayo Clin Proc 87, 643-651 (2012).

15. Shetty, N., Wren, M. W. \& Coen, P. G. The role of glutamate dehydrogenase for the detection of Clostridium difficile in faecal samples: a meta-analysis. J Hosp Infect 77, 1-6 (2011).

16. Petra, M., Constantine, G., Jonathan, D., Roger, H. \& Yemisi, T. Cochrane Handbook for Diagnostic Test Accuracy Reviews: Chapter 10 Analysing and Presenting Results (ver 1.0). (2010). Available at http://dta.cochrane.org/handbook-dta-reviews. Accessed on December 25th 2015.

17. Leeflang, M. M., Deeks, J. J., Takwoingi, Y. \& Macaskill, P. Cochrane diagnostic test accuracy reviews. Syst Rev 2, 82 (2013).

18. Leeflang, M. M., Deeks, J. J., Gatsonis, C., Bossuyt, P. M. \& Group, C. D. T. A. W. Systematic reviews of diagnostic test accuracy. Ann Intern Med 149, 889-897 (2008).

19. Harbord, R. M. et al. An empirical comparison of methods for meta-analysis of diagnostic accuracy showed hierarchical models are necessary. J Clin Epidemiol 61, 1095-1103 (2008).

20. Booth, A. et al. The nuts and bolts of PROSPERO: an international prospective register of systematic reviews. Syst Rev 1, 2 (2012).

21. Moher, D., Liberati, A., Tetzlaff, J., Altman, D. G. \& Group, P. Preferred reporting items for systematic reviews and meta-analyses: the PRISMA statement. BMJ 339, b2535 (2009).

22. Whiting, P. F. et al. QUADAS-2: a revised tool for the quality assessment of diagnostic accuracy studies. Ann Intern Med 155, 529-536 (2011)

23. Planche, T. D. et al. Differences in outcome according to Clostridium difficile testing method: a prospective multicentre diagnostic validation study of C difficile infection. Lancet Infect Dis 13, 936-945 (2013).

24. Doebler, P. Package 'mada'. Available from: https://cran.r-project.org/web/packages $/ \mathrm{mada} / \mathrm{mada} . \mathrm{pdf} \#$ search='mada+r'. Accessed on November 5th, 2015.

25. Doebler P. \& Holling H. Meta-analysis of diagnostic accuracy with mada. Available from: https://cran.r-project.org/web/packages/ mada/vignettes/mada.pdf\#search='mada+r+systematic+review+diagnostic+accuracy'. Accessed on November 5th, 2015.

26. Jones, C. M. \& Athanasiou, T. Summary receiver operating characteristic curve analysis techniques in the evaluation of diagnostic tests. Ann Thorac Surg 79, 16-20 (2005).

27. Grimes, D. A. \& Schulz, K. F. Refining clinical diagnosis with likelihood ratios. Lancet 365, 1500-1505 (2005).

28. Schünemann, H. J. et al. Grading quality of evidence and strength of recommendations for diagnostic tests and strategies. BMJ 336, $1106-1110$ (2008).

29. Higgins, P. J. \& Green, S. Cochrane Handbook for Systematic Reviews of Interventions (Version 5.1.0). (2011). Available from: http://handbook.cochrane.org/front_page.htm. Accessed on December 4th, 2015.

30. GRADE working group. GRADE. Available from: http://www.gradeworkinggroup.org/. Accessed on May 20 th, 2016.

31. Larson, A. M., Fung, A. M. \& Fang, F. C. Evaluation of tcdB real-time PCR in a three-step diagnostic algorithm for detection of toxigenic Clostridium difficile. J Clin Microbiol 48 (2010).

32. Jensen, M. B. et al. Diagnosis of Clostridium difficile: real-time PCR detection of toxin genes in faecal samples is more sensitive compared to toxigenic culture. Eur J Clin Microbiol Infect Dis 34, 727-736 (2015). 


\section{Author Contributions}

J.A. contributed for study search, quality check, data extraction, and drafting. H.N. work for study search, quality check, data extraction, and analysis as a principal investigator. A.F., T.H. and H.O. contributed for study conception. S.K., T.N. critically revised the manuscript. T.K. worked as methodologist. A.N. coordinated the study. All authors reviewed and finally approved the manuscript.

\section{Additional Information}

Supplementary information accompanies this paper at http://www.nature.com/srep

Competing financial interests: The authors declare no competing financial interests.

How to cite this article: Arimoto, J. et al. Diagnostic test accuracy of glutamate dehydrogenase for Clostridium difficile: Systematic review and meta-analysis. Sci. Rep. 6, 29754; doi: 10.1038/srep29754 (2016).

(c) (i) This work is licensed under a Creative Commons Attribution 4.0 International License. The images or other third party material in this article are included in the article's Creative Commons license, unless indicated otherwise in the credit line; if the material is not included under the Creative Commons license, users will need to obtain permission from the license holder to reproduce the material. To view a copy of this license, visit http://creativecommons.org/licenses/by/4.0/ 